\title{
The Modified Successive Overrelaxation Method with Fixed Parameters
}

\author{
By David R. Kincaid and David M. Young*
}

\begin{abstract}
Expressions for the spectral radius and for certain norms of the modified successive overrelaxation method with fixed parameters are derived. Also established are expressions for the virtual spectral radius and for certain virtual norms of this method. Parameter restrictions are determined so that the spectral radius and the norms coincide with the virtual spectral radius and the virtual norms, respectively. Optimum parameters which minimize these expressions are obtained. These results extend those of Young [11], [12].
\end{abstract}

1. Introduction. We consider convergence properties of the modified successive overrelaxation method with fixed parameters for solving the system of equations

$$
A u=b .
$$

Here $A$ is a real square positive definite** matrix of order $N, b$ is a real vector, and $u$ is the solution vector which is to be determined. We assume that $A$ has the form

$$
A=\left(\begin{array}{ll}
D_{1} & H_{1} \\
H_{2} & D_{2}
\end{array}\right)
$$

where $D_{1}$ and $D_{2}$ are square diagonal matrices. Letting $D$ be the diagonal matrix whose diagonal elements coincide with those of $A$, we note that

$$
B=I-D^{-1} A=\left(\begin{array}{ll}
0 & F \\
G & 0
\end{array}\right)
$$

where $F=-D_{1}^{-1} H_{1}$ and $G=-D_{2}^{-1} H_{2}$.

To define the modified successive overrelaxation method, we first write (1.1) in the form

$$
\begin{aligned}
& u_{1}=F u_{2}+c_{1}, \\
& u_{2}=G u_{1}+c_{2}
\end{aligned}
$$

with $u$ and $c=D^{-1} b$ partitioned according to the representation (1.2) of $A$. The

Received November 19, 1971.

AMS 1969 subject classifications. Primary 6535; Secondary 1538, 6565.

Key words and phrases. Modified successive overrelaxation method, fixed relaxation parameters, spectral radius, virtual spectral radius, $D^{1 / 2}$-norm, virtual $D^{1 / 2}$-norm, $A^{1 / 2}$-norm, virtual $A^{1 / 2}$-norm, relaxation parameters.

* Work on this paper was sponsored in part by NSF Grant GP-23655 and U. S. Army Research Office (Durham) Grant DA-ARO(D)-31-124-G1050 at The University of Texas at Austin.

** A real matrix $A$ is positive definite if $A$ is symmetric and if the inner product $(x, A x)>0$ for all real $x \neq 0$. 
modified successive overrelaxation (MSOR) method is given by

$$
\begin{aligned}
& u_{1}^{(m+1)}=\omega\left\{F u_{2}^{(m)}+c_{1}\right\}+(1-\omega) u_{1}^{(m)}, \\
& u_{2}^{(m+1)}=\omega^{\prime}\left\{G u_{1}^{(m+1)}+c_{2}\right\}+\left(1-\omega^{\prime}\right) u_{2}^{(m)}, \quad m \geqq 0,
\end{aligned}
$$

where $\omega$ and $\omega^{\prime}$ are fixed relaxation parameters such that $0<\omega<2$ and $0<\omega^{\prime}<2$. Evidently, we may write (1.4) in the form

$$
u^{(m+1)}=\mathfrak{L}_{\omega, \omega^{\prime}} u^{(m)}+k_{\omega, \omega^{\prime}},
$$

where

$$
\begin{aligned}
\mathfrak{L}_{\omega, \omega^{\prime}} & =\left(\begin{array}{cc}
I_{1} & 0 \\
-\omega^{\prime} G & I_{2}
\end{array}\right)^{-1}\left(\begin{array}{cc}
(1-\omega) I_{1} & \omega F \\
0 & \left(1-\omega^{\prime}\right) I_{2}
\end{array}\right) \\
& =\left(\begin{array}{cc}
(1-\omega) I_{1} & \omega F \\
(1-\omega) \omega^{\prime} G & \omega \omega^{\prime} G F+\left(1-\omega^{\prime}\right) I_{2}
\end{array}\right)
\end{aligned}
$$

and

$$
k_{\omega, \omega^{\prime}}=\left(\begin{array}{cc}
I_{1} & 0 \\
-\omega^{\prime} G & I_{2}
\end{array}\right)^{-1}\left(\begin{array}{l}
\omega c_{1} \\
\omega^{\prime} c_{2}
\end{array}\right) .
$$

A means of determining the convergence behavior of the MSOR method is obtained by the spectral radius of $\mathfrak{L}_{\omega, \omega}$, which is the modulus of the eigenvalue of largest modulus and is denoted by $S\left(\mathfrak{L}_{\omega, \omega^{\prime}}\right)$. Young and Kincaid [10] established a "basic eigenvalue relation" for $2 \times 2$ block matrices (see also Young [12]). This eigenvalue relation applied to $\mathfrak{L}_{\omega, \omega}$, implies the following.

If $\mu$ is an eigenvalue of $B$ and if $\lambda$ is an eigenvalue of

$$
M=\left(M_{i, i}\right)=\left(\begin{array}{cc}
1-\omega & \omega \mu \\
(1-\omega) \omega^{\prime} \mu & \omega \omega^{\prime} \mu^{2}+1-\omega^{\prime}
\end{array}\right),
$$

then $\lambda$ is an eigenvalue of $\mathscr{L}_{\omega, \omega^{\prime}}$. An exception is made for the case $\mu=0$ where at least one of the eigenvalues of $M$, but not necessarily both, is an eigenvalue of $\mathcal{L}_{\omega, \omega^{\prime}}$. Conversely, if $\lambda$ is an eigenvalue of $\mathfrak{L}_{\omega, \omega^{\prime}}$ then $\lambda$ is an eigenvalue of $M$ for some eigenvalue $\mu$ of $B$.

Thus, the eigenvalues of $\mathfrak{L}_{\omega, \omega^{\prime}}$ and $B$ are related by the quadratic equation $\operatorname{det}(M-\lambda I)=0$ which is of the form

$$
\lambda^{2}-t\left(\mu, \omega, \omega^{\prime}\right) \lambda+\delta\left(\omega, \omega^{\prime}\right)=0
$$

where

$$
\begin{aligned}
t\left(\mu, \omega, \omega^{\prime}\right) & =\operatorname{trace} M=M_{1,1}+M_{2,2}, \\
\delta\left(\omega, \omega^{\prime}\right) & =\operatorname{det} M=M_{1,1} M_{2,2}-M_{2,1} M_{1,2} .
\end{aligned}
$$

As in [11], we study the virtual spectral radius of $\mathfrak{L}_{\omega, \omega}$, which is given by

$$
\bar{S}\left(\mathscr{L}_{\omega, \omega^{\prime}}\right)=\max _{\mu \in \bar{S}_{B}} \gamma\left(\mu, \omega, \omega^{\prime}\right),
$$

where $\gamma\left(\mu, \omega, \omega^{\prime}\right)$ is the root radius of (1.7) for each $\mu$, i.e., the maximum of the moduli 
of the roots of (1.7) for each $\mu$. Here, $\bar{S}_{B}$ is the convex hull of the set of eigenvalues of $B$. Clearly, $S\left(\mathfrak{L}_{\omega, \omega^{\prime}}\right) \leqq \bar{S}\left(\mathfrak{L}_{\omega, \omega^{\prime}}\right)$, with equality whenever the maximum in (1.8) occurs at an eigenvalue of $B$, and such a maximum root of (1.7) is an eigenvalue of $\mathfrak{L}_{\omega, \omega^{\prime}}$ for this eigenvalue of $B$.

Other measures of convergence are based on various norms. The spectral norm of $\mathfrak{L}_{\omega, \omega^{\prime}}$ is defined by

$$
\left\|\mathfrak{L}_{\omega, \omega^{\prime}}\right\|_{2}=\left\{S\left(\mathfrak{L}_{\omega, \omega^{\prime}} \mathfrak{L}_{\omega, \omega^{\prime}}^{T}\right)\right\}^{1 / 2} .
$$

The $D^{1 / 2}$-norm and the $A^{1 / 2}$-norm of $\mathfrak{L}_{\omega, \omega^{\prime}}$ are given by

$$
\begin{aligned}
& \left\|\mathfrak{L}_{\omega, \omega^{\prime}}\right\|_{D^{1 / 2}}=\left\|D^{1 / 2} \mathscr{L}_{\omega, \omega^{\prime}} D^{-1 / 2}\right\|_{2}, \\
& \left\|\mathfrak{L}_{\omega, \omega^{\prime}}\right\|_{A^{1 / 2}}=\left\|A^{1 / 2} \mathfrak{L}_{\omega_{,} \omega^{\prime}} A^{-1 / 2}\right\|_{2} .
\end{aligned}
$$

The virtual $D^{1 / 2}$-norm and the virtual $A^{1 / 2}$-norm of $\mathfrak{L}_{\omega, \omega}$ are given by (1.10) and (1.9) with the virtual spectral radius of the appropriate matrix. We denote the virtual norm with an asterisk to the right of the norm. Clearly, $\left\|\mathfrak{L}_{\omega^{,} \omega^{\prime}}\right\|_{D^{1 / 2}} \leqq\left\|\mathfrak{L}_{\omega^{\prime} \omega^{\prime}}\right\|_{D^{1 / 2}}^{*}$ and $\left\|\mathfrak{L}_{\omega, \omega^{\prime}}\right\|_{A^{1 / 2}} \leqq\left\|\mathfrak{L}_{\omega^{\prime} \omega^{\prime}}\right\|_{A^{1 / 2}}^{*}$ with equality whenever the virtual spectral radius coincides with the spectral radius of the appropriate matrix.

When determining either the (virtual) spectral radius, (virtual) $D^{1 / 2}$-norm, or (virtual) $A^{1 / 2}$-norm, it is shown in Young and Kincaid [10] that it suffices to assume that $A$ has diagonal elements of unity and, consequently, that $A=I-B$. For more details on this and for more information on material given in this section, see Young [11], [12] and Kincaid [6], [7].

Evidently, the $D^{1 / 2}$-norm is given by $\left\|\mathscr{L}_{\omega, \omega^{\prime}}\right\|_{D^{1 / 2}}=\left\{S\left(\mathfrak{L}_{\omega, \omega^{\prime}} \mathfrak{L}_{\omega, \omega^{\prime}}^{T}\right)\right\}^{1 / 2}$. Consequently, the virtual $D^{1 / 2}$-norm is obtained from

$$
\left\|\mathfrak{L}_{\omega, \omega^{\prime}}\right\|_{D^{1 / 2}}^{*}=\max _{\mu \in \bar{S}_{B}} \Gamma^{1 / 2}(\mu),
$$

where $\Gamma(\mu)$ is the root radius for each $\mu$ of the quadratic equation

$$
\Lambda^{2}-T\left(\mu, \omega, \omega^{\prime}\right) \Lambda+\Delta\left(\omega, \omega^{\prime}\right)=0
$$

and where

$$
\begin{aligned}
T\left(\mu, \omega, \omega^{\prime}\right) & =\operatorname{trace} M M^{T}=M_{1,1}^{2}+M_{1,2}^{2}+M_{2,1}^{2}+M_{2,2}^{2}, \\
\Delta\left(\omega, \omega^{\prime}\right) & =\operatorname{det} M M^{T}=\left(M_{1,1} M_{2,2}-M_{2,1} M_{1,2}\right)^{2} .
\end{aligned}
$$

The $A^{1 / 2}$-norm is given by

$$
\begin{aligned}
\left\|\mathfrak{L}_{\omega, \omega^{\prime}}\right\|_{A^{1 / 2}} & =\left\{S\left(\mathscr{L}_{\omega, \omega^{\prime}} A^{-1} \mathfrak{L}_{\omega, \omega^{\prime}}^{T} A\right)\right\}^{1 / 2} \\
& =\left\{S\left(\mathscr{L}_{\omega, \omega^{\prime}}^{\prime}\left(\mathscr{L}_{\omega, \omega^{\prime}}^{\prime}\right)^{T}\right)\right\}^{1 / 2},
\end{aligned}
$$

where $\mathscr{L}_{\omega, \omega^{\prime}}^{\prime}=A^{1 / 2} \mathscr{L}_{\omega, \omega^{\prime}} A^{-1 / 2}$. Moreover, the virtual $A^{1 / 2}$-norm is obtained from (1.8) with the appropriate matrix from (1.13).

In Section 2, we determine an expression for the (virtual) spectral radius of $\mathfrak{L}_{\omega, \omega}$ and also convenient upper and lower bounds. The domain in the $\left(\omega, \omega^{\prime}\right)$-plane where the spectral radius and the virtual spectral radius coincide is established. These results are then used to obtain corresponding expressions and bounds for the $A^{1 / 2}$ norm.

Young [11] discussed the problem of choosing $\omega$ and $\omega^{\prime}$ to minimize the virtual 
$D^{1 / 2}$-norm of $\mathfrak{E}_{\omega, \omega^{\prime}}$. A partial solution of this problem was stated there without proof. In Section 3, the problem is solved completely. Also, an expression for the (virtual) $D^{1 / 2}$-norm is given and the region of agreement between the $D^{1 / 2}$-norm and the virtual $D^{1 / 2}$-norm is obtained. Perspective graphs are given that illustrate the behavior of the virtual spectral radius, the virtual $A^{1 / 2}$-norm, and the virtual $D^{1 / 2}$ norm.

The development of the MSOR method has resulted from the work of De Vogelaere [1], Young, et al. [9], McDowell [4], Taylor [5], Young and Kincaid [10], Young [11], Kincaid [6], [7], and, most recently, Young [12]. The theorems in this paper expand those of Young [12] on the virtual spectral radius, the virtual $A^{1 / 2}$-norm, and the virtual $D^{1 / 2}$-norm. Interest in this area has been expressed in recent papers by Ehrlich [2], [3] where the MSOR method with fixed parameters is used for solving the biharmonic equation as a coupled set of finite-difference equations.

2. Spectral Radius and $A^{1 / 2}$-Norm. We now determine an expression for the (virtual) spectral radius of $\mathcal{L}_{\omega, \omega^{\prime}}$ and determine when the spectral radius and the virtual spectral radius coincide. Note that this theorem is implied by the discussion given by Young [12].

THEOREM 2.1. If $A$ is a positive definite matrix of the form (1.2), if $0<\omega<2$ and $0<\omega^{\prime}<2$, and

(i) if $\omega+\omega^{\prime}-\frac{1}{2} \bar{\mu}^{2} \omega \omega^{\prime} \leqq 2$, then

$$
S\left(\mathscr{L}_{\omega, \omega^{\prime}}\right)=\bar{S}\left(\mathfrak{L}_{\omega, \omega^{\prime}}\right)=\sigma\left(\omega, \omega^{\prime}\right)+\left\{\sigma^{2}\left(\omega, \omega^{\prime}\right)-(\omega-1)\left(\omega^{\prime}-1\right)\right\}^{1 / 2}
$$

where

$$
\sigma\left(\omega, \omega^{\prime}\right)=\frac{1}{2}\left|2-\omega-\omega^{\prime}+\omega \omega^{\prime} \bar{\mu}^{2}\right|,
$$

(ii) if $\omega+\omega^{\prime}-\frac{1}{2} \bar{\mu}^{2} \omega \omega^{\prime} \geqq 2$, then

$$
\begin{aligned}
\bar{S}\left(\mathscr{L}_{\omega, \omega^{\prime}}\right) & =\omega-1, & & \omega \geqq \omega^{\prime}, \\
& =\omega^{\prime}-1, & & \omega \leqq \omega^{\prime} .
\end{aligned}
$$

Proof. From Section 1, the virtual spectral radius of $\mathcal{L}_{\omega, \omega^{\prime}}$ is the maximum root radius of the characteristic Eq. (1.7) over the interval $-\bar{\mu} \leqq \mu \leqq \bar{\mu}$ where $\bar{\mu}=S(B)$. Evidently,

$$
\begin{aligned}
t\left(\mu, \omega, \omega^{\prime}\right) & =2-\omega-\omega^{\prime}+\omega \omega^{\prime} \mu^{2}, \\
\delta\left(\omega, \omega^{\prime}\right) & =(\omega-1)\left(\omega^{\prime}-1\right) .
\end{aligned}
$$

Notice that this quadratic equation can also be written in the form

$$
(\lambda+\omega-1)\left(\lambda+\omega^{\prime}-1\right)=\omega \omega^{\prime} \mu^{2} \lambda .
$$

The maximum root radius of (1.7) for fixed $\omega$ and $\omega^{\prime}$ is found by maximizing $\left|t\left(\mu, \omega, \omega^{\prime}\right)\right|$ over the interval $-\bar{\mu} \leqq \mu \leqq \bar{\mu}$ (see Young [11] for details). Since $t(\mu) \equiv t\left(\mu, \omega, \omega^{\prime}\right)$ is a linear function of $\mu^{2}$, we have

$$
\max _{-\bar{\mu} \leqq \mu \leqq \bar{\mu}}|t(\mu)|=\max \{|t(0)|,|t(\bar{\mu})|\} .
$$

We note by Fig. 2.1 that in the $\left(\omega, \omega^{\prime}\right)$-plane there are two pertinent regions since $\omega$ 


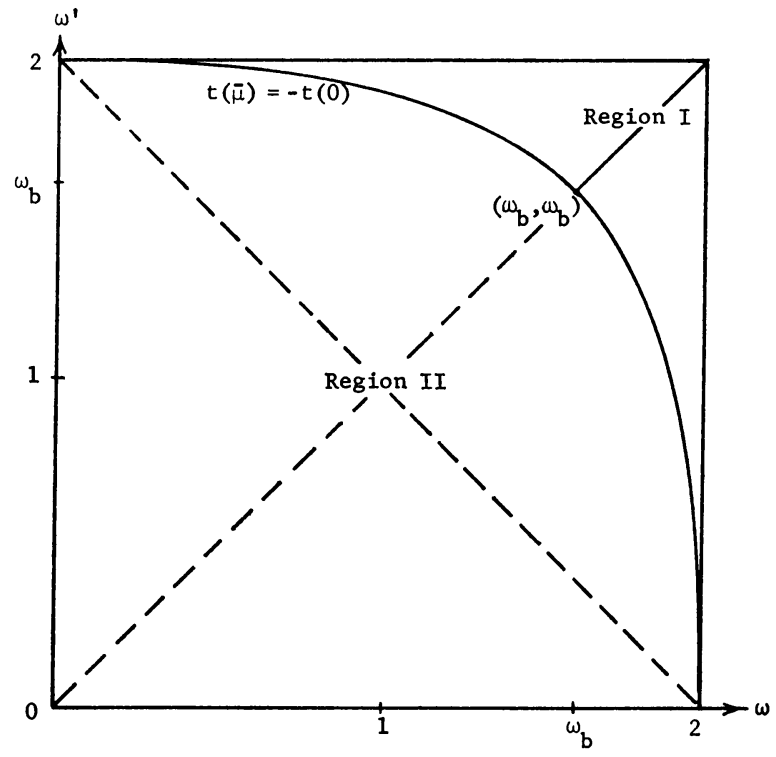

Figure 2.1

and $\omega^{\prime}$ are fixed between zero and two. We let Region I and Region II be such that $t(\bar{\mu}) \leqq-t(0)$ and $t(\bar{\mu}) \geqq-t(0)$, respectively.

Consequently, we have

$$
\begin{array}{rlrl}
\max _{-\bar{\mu} \leqq \mu \leqq \bar{\mu}}\left|t\left(\mu, \omega, \omega^{\prime}\right)\right| & =\left|t\left(\bar{\mu}, \omega, \omega^{\prime}\right)\right|, \quad & \left(\omega, \omega^{\prime}\right) \in \text { Region II }, \\
& =-t\left(0, \omega, \omega^{\prime}\right), \quad\left(\omega, \omega^{\prime}\right) \in \text { Region I. }
\end{array}
$$

Since $\bar{\mu}$ is necessarily an eigenvalue of $B$, we have established (2.1) in Region II; however, since zero is not necessarily an eigenvalue of $B$, we can establish only an expression for the virtual spectral radius in Region $I$.

We now prove

THEOREM 2.2. If $A$ is a positive definite matrix of the form (1.2), then, for $\omega+\omega^{\prime}-$ $\frac{1}{2} \bar{\mu}^{2} \omega \omega^{\prime} \leqq 2,0<\omega<2$, and $0<\omega^{\prime}<2$, we have

$$
\min _{\omega, \omega^{\prime}} S\left(\mathfrak{L}_{\omega_{,} \omega^{\prime}}\right)=S\left(\mathfrak{L}_{\omega_{b}, \omega_{b}}\right)=\omega_{b}-1 \text {. }
$$

Moreover,

$$
\min _{0<\omega<2 ; 0<\omega^{\prime}<2} \bar{S}\left(\mathscr{L}_{\omega, \omega^{\prime}}\right)=\bar{S}\left(\mathfrak{L}_{\omega_{b}, \omega_{b}}\right)=\omega_{b}-1,
$$

where $\omega_{b}=2 /\left(1+\left(1-\bar{\mu}^{2}\right)^{1 / 2}\right)$. Also, for $0<\omega \leqq 1$ and $0<\omega^{\prime} \leqq 1$, we have

$$
1-\left(\omega \omega^{\prime}\right)^{1 / 2}\left(1-\bar{\mu}^{2}\right) \leqq S\left(\mathfrak{L}_{\omega, \omega^{\prime}}\right)=\bar{S}\left(\mathfrak{L}_{\omega, \omega^{\prime}}\right) \leqq 1-\omega \omega^{\prime}\left(1-\bar{\mu}^{2}\right) .
$$

Proof. From Young [11], [12], we have (2.6) and, consequently, (2.5) by (2.1).

The spectral radius of $\mathfrak{L}_{\omega, \omega^{\prime}}$, for $0<\omega \leqq 1$ and $0<\omega^{\prime} \leqq 1$, is the root radius of

$$
\lambda^{2}-\left[2-\omega-\omega^{\prime}+\omega \omega^{\prime} \bar{\mu}^{2}\right] \lambda+(\omega-1)\left(\omega^{\prime}-1\right)=0,
$$

where $0<\bar{\mu}<1$. Letting $\theta=1-\lambda$, we have

$$
\theta^{2}-\left[\omega+\omega^{\prime}-\omega \omega^{\prime} \bar{\mu}^{2}\right] \theta+\omega \omega^{\prime}\left(1-\bar{\mu}^{2}\right)=0 .
$$


Next, letting $\phi=\theta-\omega \omega^{\prime}\left(1-\bar{\mu}^{2}\right)$, we have

$$
\phi^{2}-\left[\omega+\omega^{\prime}-\omega \omega^{\prime}\left(2-\bar{\mu}^{2}\right)\right] \phi+\omega \omega^{\prime}(\omega-1)\left(\omega^{\prime}-1\right)\left(1-\bar{\mu}^{2}\right)=0 .
$$

Since $\omega+\omega^{\prime}-\omega \omega^{\prime}\left(2-\bar{\mu}^{2}\right) \geqq \omega^{2}+\left(\omega^{\prime}\right)^{2}-2 \omega \omega^{\prime} \geqq 0$ and since the roots of $(2.10)$ are real, both roots of $(2.10)$ are nonnegative. Hence, the right-hand inequality of (2.7) follows. The smaller root of (2.9) is given by

$$
\theta=2 \omega \omega^{\prime}\left(1-\bar{\mu}^{2}\right)\left[w+\left(w^{2}-4 \omega \omega^{\prime}\left(1-\bar{\mu}^{2}\right)\right)^{1 / 2}\right]^{-1}
$$

where $w=\omega+\omega^{\prime}-\omega \omega^{\prime} \bar{\mu}^{2}$. Consider $\theta-\left(\omega \omega^{\prime}\right)^{1 / 2}\left(1-\bar{\mu}^{2}\right)=\left(\omega \omega^{\prime}\right)^{1 / 2}\left(1-\bar{\mu}^{2}\right) R$ where $R=2\left(\omega \omega^{\prime}\right)^{1 / 2}\left[v+\left(v^{2}-4 \omega \omega^{\prime}\left(1-\bar{\mu}^{2}\right)\right)^{1 / 2}\right]^{-1}-1$ with $v=\left(\omega^{\prime} / \omega\right)^{1 / 2}+$ $\left(\omega / \omega^{\prime}\right)^{1 / 2}-\bar{\mu}^{2}\left(\omega \omega^{\prime}\right)^{1 / 2}$. But $v \geqq 2-\bar{\mu}^{2}$, since $\left(\omega^{\prime} / \omega\right)^{1 / 2}+\left(\omega / \omega^{\prime}\right)^{1 / 2} \geqq 2$ and $\left(\omega \omega^{\prime}\right)^{1 / 2} \leqq 1$. Therefore, $R \leqq 0$ and $\theta \leqq\left(\omega \omega^{\prime}\right)^{1 / 2}\left(1-\bar{\mu}^{2}\right)$. Consequently, the left-hand inequality of (2.7) holds.

The result (2.6) was proven by Young, et al. [9]. The second inequality of (2.7) is stronger than a similar result which was stated by Young [11, p. 80], [12, p. 282] and for which an incorrect proof was given.

Fig. 2.2 illustrates the behavior of $\bar{S}\left(\mathfrak{L}_{\omega, \omega^{\prime}}\right)$ for $0 \leqq \omega \leqq 2$ and $0 \leqq \omega^{\prime} \leqq 2$. The point of the partially hidden cusp in the perspective graph corresponds to the value of the virtual spectral radius at $\left(\omega_{b}, \omega_{b}\right)$. Notice that the virtual spectral radius is a positive function of $\omega$ and $\omega^{\prime}$ with the value unity at the boundary of the square $0 \leqq \omega \leqq 2$ and $0 \leqq \omega^{\prime} \leqq 2$.
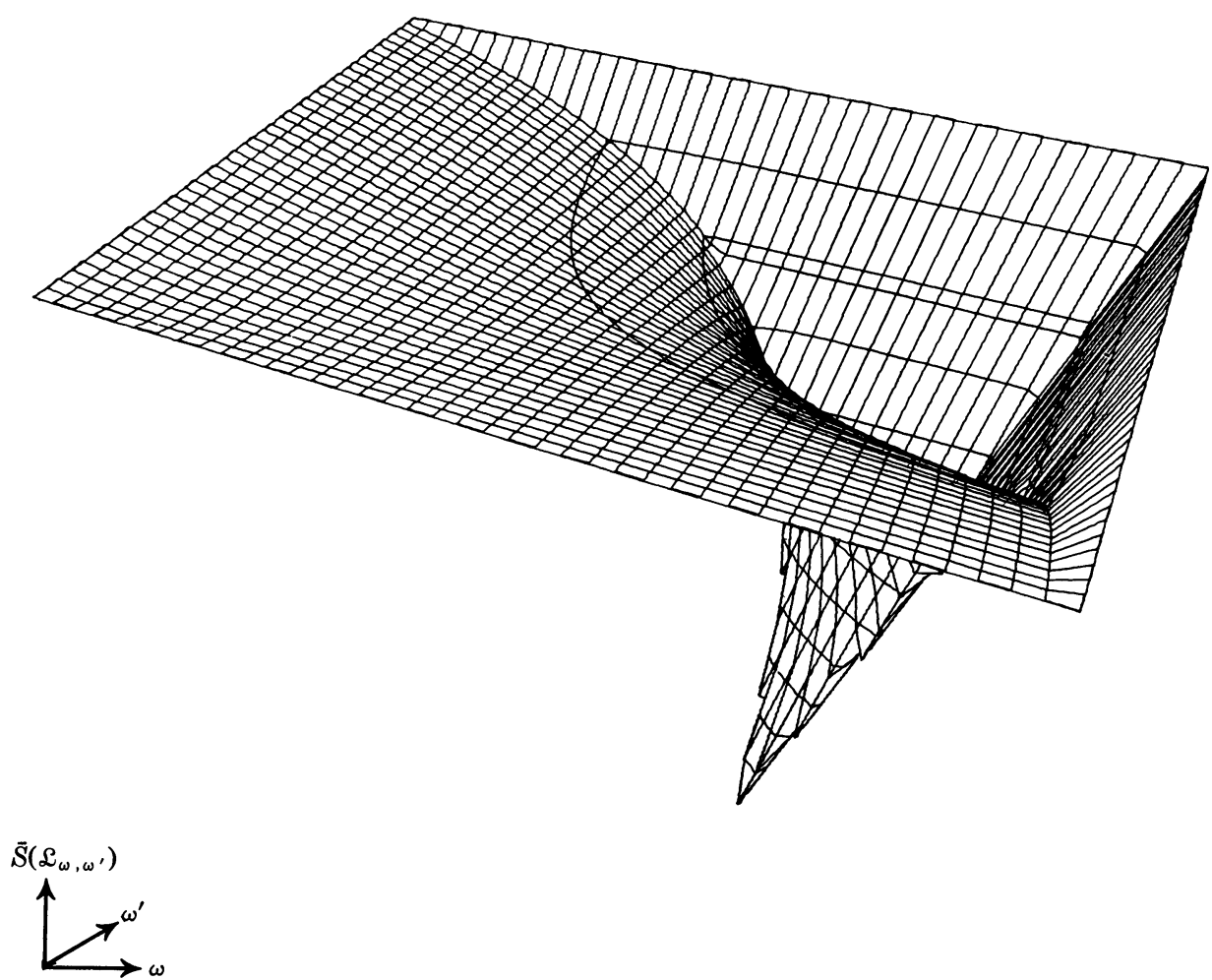

FIGURE 2.2. Virtual Spectral Radius of $\mathfrak{L}_{\omega, \omega}$. 
We now determine the (virtual) $A^{1 / 2}$-norm of $\mathfrak{L}_{\omega, \omega^{\prime}}$. From Young [11], [12], we have

$$
S\left(\mathscr{L}_{\omega^{\prime}, \omega^{\prime}}^{\prime}\left(\mathscr{L}_{\omega^{\prime}, \omega^{\prime}}^{\prime}\right)^{T}\right)=S\left(\mathscr{L}_{\omega\left(2-\omega^{\prime}, \omega^{\prime}\left(2-\omega^{\prime}\right)\right.}\right),
$$

so that we obtain the following from (1.13), since $0<\omega(2-\omega) \leqq 1$ and $0<\omega^{\prime}\left(2-\omega^{\prime}\right) \leqq 1$.

THEOREM 2.3. If $A$ is a positive definite matrix of the form (1.2), and if $0<\omega<2$ and $0<\omega^{\prime}<2$, then

$$
\left\|\mathfrak{L}_{\omega, \omega^{\prime}}\right\|_{A^{1 / 2}}=\left\|\mathscr{L}_{\omega, \omega^{\prime}}\right\|_{A^{1 / 2}}^{*}=\left\{S\left(\mathscr{L}_{\omega(2-\omega), \omega^{\prime}\left(2-\omega^{\prime}\right)}\right)\right\}^{1 / 2}
$$

and

$$
\begin{aligned}
& \left\{1-\omega \omega^{\prime}(2-\omega)\left(2-\omega^{\prime}\right)\left(1-\bar{\mu}^{2}\right)\right\}^{1 / 2} \\
& \quad \leqq\left\|\mathfrak{L}_{\omega, \omega^{\prime}}\right\|_{A^{1 / 2}}=\left\|\mathfrak{L}_{\omega, \omega^{\prime}}\right\|_{A^{1 / 2}}^{*} \leqq 1-\frac{1}{2} \omega \omega^{\prime}(2-\omega)\left(2-\omega^{\prime}\right)\left(1-\bar{\mu}^{2}\right) .
\end{aligned}
$$

Also,

$$
\begin{aligned}
\min _{0<\omega<2 ; 0<\omega^{\prime}<2} & \left\|\mathscr{L}_{\omega, \omega^{\prime}}\right\|_{A^{1 / 2}} \\
= & \min _{0<\omega<2 ; 0<\omega^{\prime}<2}\left\|\mathscr{L}_{\omega, \omega^{\prime}}\right\|_{A^{1 / 2}}^{*}=\left\|\mathscr{L}_{1,1}\right\|_{A^{1 / 2}}^{*}=\left\|\mathscr{L}_{1,1}\right\|_{A^{1 / 2}}=\bar{\mu} .
\end{aligned}
$$

Fig. 2.3 illustrates the behavior of the (virtual) $A^{1 / 2}$-norm of $\mathfrak{L}_{\omega, \omega^{\prime}}$ for $0 \leqq \omega \leqq 2$ and $0 \leqq \omega^{\prime} \leqq 2$.
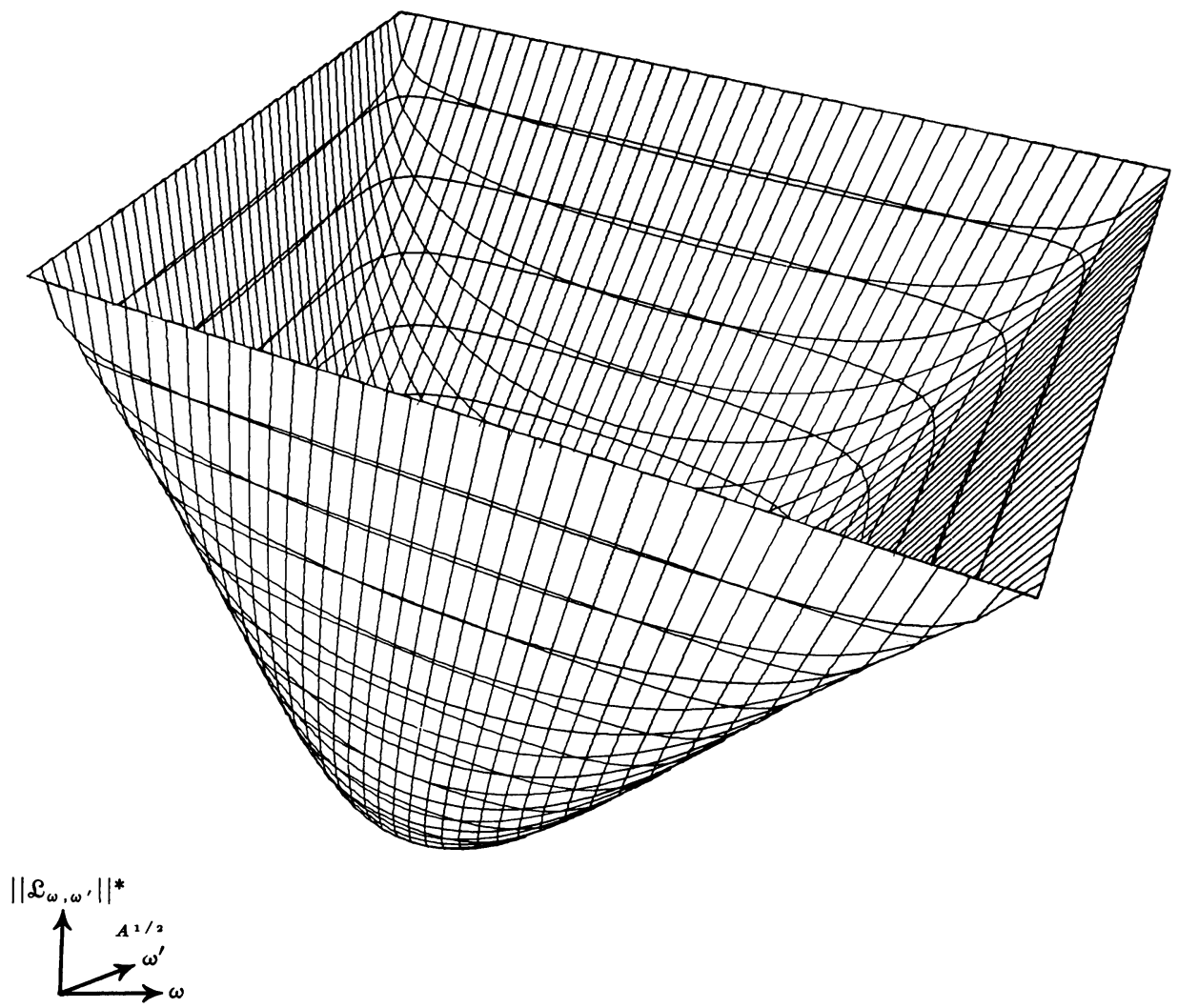

FIGURE 2.3. $A^{1 / 2-n o r m}$ of $\mathfrak{L}_{\omega, \omega^{\prime}}$. 
3. $D^{1 / 2}$-Norm. We now consider the problem of determining the (virtual) $D^{1 / 2}$-norm of $\mathscr{L}_{\omega, \omega^{\prime}}$ and the values of $\omega$ and $\omega^{\prime}$ which minimizes the (virtual) $D^{1 / 2}$. norm.

THEOREM 3.1. If $A$ is a positive definite matrix of the form (1.2), if $0<\omega<2$ and $0<\omega^{\prime}<2$, and

(i) if $\omega^{2}+\left(\omega^{\prime}\right)^{2}+\left(1+\bar{\mu}^{2}\right)\left(\omega \omega^{\prime}\right)^{2}+2 \omega \omega^{\prime}\left(1-2 \omega^{\prime}\right) \geqq 0$, then

$$
\begin{aligned}
\left\|\mathscr{L}_{\omega, \omega^{\prime}}\right\|_{D^{1 / 2}} & =\left\|\mathscr{L}_{\omega, \omega^{\prime}}\right\| \|_{D^{1 / 2}}^{*} \\
& =\psi\left(\bar{\mu}, \omega, \omega^{\prime}\right)+\left\{\psi^{2}\left(\bar{\mu}, \omega, \omega^{\prime}\right)+\mid(\omega-1)\left(\omega^{\prime}-1\right)\right\}^{1 / 2},
\end{aligned}
$$

where

$$
\begin{aligned}
& \psi\left(\bar{\mu}, \omega, \omega^{\prime}\right) \\
& =\frac{1}{2}\left\{\left[|\omega-1|-\left|\omega^{\prime}-1\right|\right]^{2}+\left[\left(\omega+\omega^{\prime}\right)^{2}-\omega\left(\omega^{\prime}\right)^{2}(4-\omega)\right] \bar{\mu}^{2}+\left(\omega \omega^{\prime}\right)^{2} \bar{\mu}^{4}\right\}^{1 / 2} \text {, }
\end{aligned}
$$

$$
\left\|\mathscr{L}_{\omega, \omega^{\prime}}\right\|_{D^{1 / 2}}^{*}=\omega^{\prime}-1 \text {. }
$$

Proof. From Section 1, the virtual $D^{1 / 2}$-norm of $\mathfrak{L}_{\omega, \omega}$ is given by (1.11). For notational simplicity, we let $x=\omega$ and $y=\omega^{\prime}$. From (1.12) and (1.6), we have

$$
\begin{aligned}
T(\mu, x, y) & =(1-x)^{2}+(1-x)^{2} y^{2} \mu^{2}+x^{2} \mu^{2}+\left(x y \mu^{2}+1-y\right)^{2}, \\
\Delta(x, y) & =(1-x)^{2}(1-y)^{2} .
\end{aligned}
$$

For fixed $x$ and $y$ such that $0<x<2$ and $0<y<2$, we observe that, for $T(\mu) \equiv$ $T(\mu, x, y)$,

$$
\max _{-\bar{\mu} \leq \mu \leq \bar{\mu}}|T(\mu)|=\max \{T(0), T(\bar{\mu})\} .
$$

By Fig. 3.1, in the $(x, y)$-plane there are two pertinent regions.

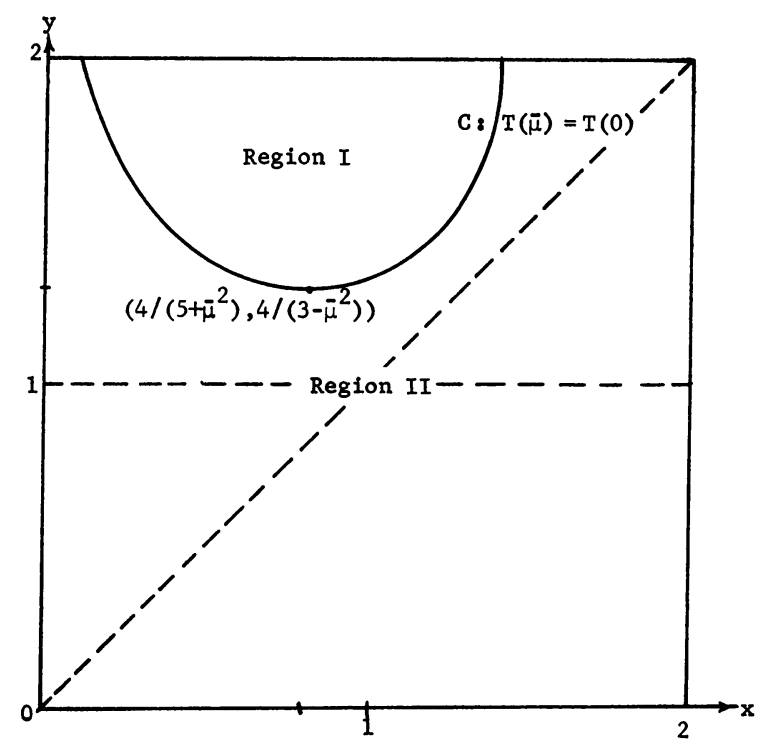

FIGURE 3.1 
We let Region I and Region II be such that $T(\bar{\mu}) \leqq T(0)$ and $T(\bar{\mu}) \geqq T(0)$, respectively. It is easy to show that $T(\bar{\mu})=T(0)$ if and only if

$$
x^{2}\left(\bar{\mu}^{2} y^{2}+y^{2}+1\right)+x\left(2 y-4 y^{2}\right)+y^{2}=0 .
$$

Clearly, we have

$$
\begin{aligned}
\max _{-\bar{\mu} \leqq \mu \leqq \bar{\mu}}|T(\mu, x, y)| & =T(\bar{\mu}, x, y), \quad(x, y) \in \text { Region II }, \\
& =T(0, x, y), \quad(x, y) \in \text { Region I } .
\end{aligned}
$$

Since

$$
\Gamma^{1 / 2}=\frac{1}{2}(T-2 \sqrt{ } \Delta)^{1 / 2}+\frac{1}{2}(T+2 \sqrt{ } \Delta)^{1 / 2},
$$

the proof follows from (3.4), (3.6), and (3.7). Clearly, the $D^{1 / 2}$-norm and the virtual $D^{1 / 2}$-norm coincide in Region II.

We now determine the values of $\omega$ and $\omega^{\prime}$ which minimize the (virtual) $D^{1 / 2}$-norm of $\mathfrak{L}_{\omega, \omega^{\prime}}$.

THEOREM 3.2. If $A$ is a positive definite matrix of the form (1.2) and

(i) if $0<\bar{\mu} \leqq(1 / 3)^{1 / 2}$ then

$$
\min _{0<\omega<2 ; 0<\omega^{\prime}<2}\left\|\mathfrak{L}_{\omega, \omega^{\prime}}\right\|_{D^{1 / 2}}=\left\|\mathfrak{L}_{\omega_{0}, \omega_{0}}\right\|_{D^{1 / 2}}=\left(1-\omega_{0}\right)^{1 / 2},
$$

and

$$
\min _{0<\omega<2 ; 0<\omega^{\prime}<2}\left\|\mathfrak{L}_{\omega, \omega^{\prime}}\right\|_{D^{1 / 2}}^{*}=\left\|\mathfrak{L}_{\omega_{0}, \omega_{0}}\right\|_{D^{1 / 2}}^{*}=\left(1-\omega_{0}\right)^{1 / 2}=\bar{\mu} /\left(1+\bar{\mu}^{2}\right)^{1 / 2},
$$

where $\omega_{0}=1 /\left(1+\bar{\mu}^{2}\right), \omega_{0}^{\prime}=1 /\left(1-\bar{\mu}^{2}\right)$,

(ii) if $(1 / 3)^{1 / 2} \leqq \bar{\mu}<1$, then

$$
\begin{aligned}
\min _{0<\omega<2 ; 0<\omega^{\prime}<2}\left\|\mathscr{L}_{\omega, \omega^{\prime}}\right\|_{D^{1 / 2}}^{*} & =\left\|\mathfrak{L}_{\omega_{0}, \omega_{0}}\right\|_{D^{1 / 2}}^{*} \\
& =\left\|\mathfrak{L}_{\omega_{0}, \omega_{0}}\right\|_{D^{1 / 2}}=\omega_{0}^{\prime}-1=\left(1+\bar{\mu}^{2}\right) /\left(3-\bar{\mu}^{2}\right),
\end{aligned}
$$

where $\omega_{0}=4 /\left(5+\bar{\mu}^{2}\right), \omega_{0}^{\prime}=4 /\left(3-\bar{\mu}^{2}\right)$.

Proof. As before, we let $x=\omega$ and $y=\omega^{\prime}$. We now establish the minimum point for the virtual $D^{1 / 2}$-norm when restricted to Region I. By (3.3), the point which minimizes the virtual $D^{1 / 2}$-norm in Region I lies on the boundary $C$ between Region I and Region II. In order to lie on $C$ such a point must satisfy Eq. (3.6) which has two, one, or no real roots depending on whether the discriminant $4 y^{3}\left[\left(3-\bar{\mu}^{2}\right) y-4\right]$ of (3.6) is positive, zero, or negative, respectively. Thus, as indicated in Fig. 3.1, if $y>4 /\left(3-\bar{\mu}^{2}\right)$ then there are two real roots of $(3.6)$, and if $y=4 /\left(3-\bar{\mu}^{2}\right)$, then there is only one, namely, $x=4 /\left(5+\bar{\mu}^{2}\right)$. Notice that the virtual $D^{1 / 2}$-norm on the boundary $y=2$ of Region $\mathrm{I}$ is unity by (3.3). Since by (3.3) the virtual $D^{1 / 2}$-norm is independent of $x$ and since at $y=4 /\left(3-\bar{\mu}^{2}\right)$ and $x=4 /\left(5+\bar{\mu}^{2}\right)$ the virtual $D^{1 / 2}$ norm is less than unity, it follows that the optimum relaxation parameters with regard to the virtual $D^{1 / 2}$-norm are $x=4 /\left(5+\bar{\mu}^{2}\right)$ and $y=4 /\left(3-\bar{\mu}^{2}\right)$ when restricted to Region I.

Next, we seek the minimum values of $x$ and $y$ for the $D^{1 / 2}$-norm when restricted to Region II. We note that, from (1.12) and (3.8),

$$
2 \Gamma^{1 / 2}=R_{1}+R_{2},
$$


where

$$
\begin{aligned}
& R_{1}^{2}=\left(M_{1,1}-M_{2,2}\right)^{2}+\left(M_{1,2}+M_{2,1}\right)^{2}, \\
& R_{2}^{2}=\left(M_{1,1}+M_{2,2}\right)^{2}+\left(M_{1,2}-M_{2,1}\right)^{2} .
\end{aligned}
$$

Clearly, by differentiating with respect to either $x$ or $y$, we have

$$
\begin{aligned}
2\left(\Gamma^{1 / 2}\right)^{\prime}= & {\left[\left(M_{1,1}-M_{2,2}\right)\left(M_{1,1}^{\prime}-M_{2,2}^{\prime}\right)+\left(M_{1,2}+M_{2,1}\right)\left(M_{1,2}^{\prime}+M_{2,1}^{\prime}\right)\right] / R_{1} } \\
& +\left[\left(M_{1,1}+M_{2,2}\right)\left(M_{1,1}^{\prime}+M_{2,2}^{\prime}\right)+\left(M_{1,2}-M_{2,1}\right)\left(M_{1,2}^{\prime}-M_{2,1}^{\prime}\right)\right] / R_{2},
\end{aligned}
$$

whenever $R_{1} \neq 0$ and $R_{2} \neq 0$. By defining

$$
\begin{aligned}
& \theta_{1}=\tan ^{-1}\left(\frac{M_{1,2}+M_{2,1}}{M_{1,1}-M_{2,2}}\right), \\
& \theta_{2}=\tan ^{-1}\left(\frac{M_{1,2}-M_{2,1}}{M_{1,1}+M_{2,2}}\right),
\end{aligned}
$$

we have

$$
\begin{aligned}
\left(\Gamma^{1 / 2}\right)^{\prime}= & \sin \frac{1}{2}\left(\theta_{1}-\theta_{2}\right)\left\{M_{2,2}^{\prime} \sin \frac{1}{2}\left(\theta_{1}+\theta_{2}\right)+M_{2,1}^{\prime} \cos \frac{1}{2}\left(\theta_{1}+\theta_{2}\right)\right\} \\
& +\cos \frac{1}{2}\left(\theta_{1}-\theta_{2}\right)\left\{M_{1,1}^{\prime} \cos \frac{1}{2}\left(\theta_{1}+\theta_{2}\right)+M_{1,2}^{\prime} \sin \frac{1}{2}\left(\theta_{1}+\theta_{2}\right)\right\} .
\end{aligned}
$$

From (1.6) it is easy to show that

$\partial \Gamma^{1 / 2} / \partial x=\left\{\bar{\mu} \sin \frac{1}{2}\left(\theta_{1}+\theta_{2}\right)-\cos \frac{1}{2}\left(\theta_{1}+\theta_{2}\right)\right\}\left\{y \bar{\mu} \sin \frac{1}{2}\left(\theta_{1}-\theta_{2}\right)+\cos \frac{1}{2}\left(\theta_{1}-\theta_{2}\right)\right\}$ and

$\partial \Gamma^{1 / 2} / \partial y=\left\{\sin \frac{1}{2}\left(\theta_{1}-\theta_{2}\right)\right\}\left\{\left(x \bar{\mu}^{2}-1\right) \sin \frac{1}{2}\left(\theta_{1}+\theta_{2}\right)+(1-x) \bar{\mu} \cos \frac{1}{2}\left(\theta_{1}+\theta_{2}\right)\right\}$.

Notice that $\bar{\mu}$ is used, since we are restricted to Region II with $\Gamma^{1 / 2}(\bar{\mu})$ from (3.1). Thus, we have $\partial \Gamma^{1 / 2} / \partial x=0$ if at least one of the following conditions holds

$$
\begin{aligned}
& \tan \frac{1}{2}\left(\theta_{1}+\theta_{2}\right)=1 / \bar{\mu}, \\
& \tan \frac{1}{2}\left(\theta_{1}-\theta_{2}\right)=-1 /(y \bar{\mu})
\end{aligned}
$$

and $\partial \Gamma^{1 / 2} / \partial y=0$ if at least one of the following conditions holds

$$
\begin{aligned}
& \tan \frac{1}{2}\left(\theta_{1}+\theta_{2}\right)=(1-x) \bar{\mu} /\left(1-x \bar{\mu}^{2}\right), \\
& \tan \frac{1}{2}\left(\theta_{1}-\theta_{2}\right)=0 .
\end{aligned}
$$

Clearly, (3.15) and (3.13) cannot hold simultaneously, since $0<y<2$ and $0<\bar{\mu}<1$. Moreover, (3.12) and (3.14) are not simultaneously satisfied, since $\bar{\mu} \neq 1$. Hence, $\partial \Gamma^{1 / 2} / \partial x$ and $\partial \Gamma^{1 / 2} / \partial y$ vanish simultaneously when and only when at least one of the following two sets of conditions hold

$$
\begin{aligned}
& \tan \frac{1}{2}\left(\theta_{1}+\theta_{2}\right)=(1-x) \bar{\mu} /\left(1-x \bar{\mu}^{2}\right), \\
& \tan \frac{1}{2}\left(\theta_{1}-\theta_{2}\right)=-1 /(y \bar{\mu}), \\
& \tan \frac{1}{2}\left(\theta_{1}+\theta_{2}\right)=1 / \bar{\mu}, \\
& \tan \frac{1}{2}\left(\theta_{1}-\theta_{2}\right)=0 .
\end{aligned}
$$

First, we suppose that (3.16) holds. Using (3.11) together with (3.16), we have, 
from (1.6) and from the fact that $\theta_{1}=\frac{1}{2}\left(\theta_{1}+\theta_{2}\right)+\frac{1}{2}\left(\theta_{1}-\theta_{2}\right)$,

$$
\left(1-\bar{\mu}^{2}\right) x-\left(1+\bar{\mu}^{2}\right) y+2 \bar{\mu}^{2} x y=0 .
$$

Similarly, using the fact that $\theta_{2}=\frac{1}{2}\left(\theta_{1}+\theta_{2}\right)-\frac{1}{2}\left(\theta_{1}-\theta_{2}\right)$, we have

$$
\left(1+\bar{\mu}^{2}\right) x+\left(1-\bar{\mu}^{2}\right) y=2 \text {. }
$$

Solving (3.18) and (3.19) simultaneously, we find that the roots $z_{i}=\left(x_{i}, y_{i}\right)$ are $z_{1}=(1,1)$ and $z_{2}=\left(1 / \bar{\mu}^{2},-1 / \bar{\mu}^{2}\right)$. Clearly, $z_{2}$ is not allowable, since $0<y<2$. We shall show later that $z_{1}$ is not the minimum point.

Now suppose that (3.17) holds. The second equation of (3.17) implies that $\theta_{1}=$ $\theta_{2}+2 k \pi$. Hence, from (3.11), we have $M_{1,1} M_{2,1}+M_{1,2} M_{2,2}=0$, i.e., from (1.6),

$$
(1-x)^{2} y+x^{2} y \bar{\mu}^{2}+x(1-y)=0 .
$$

Moreover, $\theta_{1}=\theta_{2}+2 k \pi$ and the first equation of (3.17) implies that $\tan \theta_{1}=1 / \bar{\mu}$, i.e., from (3.11) and (1.6),

$$
\left(1+\bar{\mu}^{2}\right) x=\left(1-\bar{\mu}^{2}\right) y .
$$

Similarly, we have $\tan \theta_{2}=1 / \bar{\mu}$, i.e.,

$$
\left(1+\bar{\mu}^{2}\right) x+\left(1-\bar{\mu}^{2}\right) y=2 .
$$

Eliminating $y$ from (3.20) and (3.21), we obtain

$$
\left(1+\bar{\mu}^{2}\right)^{2} x^{3}-3\left(1+\bar{\mu}^{2}\right) x^{2}+2 x=0
$$

which has roots $z_{0}=\left(1 /\left(1+\bar{\mu}^{2}\right), 1 /\left(1-\bar{\mu}^{2}\right)\right), z_{3}=(0,0)$, and $z_{4}=\left(2 /\left(1+\bar{\mu}^{2}\right)\right.$, $\left.2 /\left(1-\bar{\mu}^{2}\right)\right)$. Clearly, $z_{3}$ and $z_{4}$ are not allowable, since $0<y<2$. Eliminating $y$ between (3.20) and (3.22), we obtain

$$
\left(1+\bar{\mu}^{2}\right)^{2} x^{3}-5\left(1+\bar{\mu}^{2}\right) x^{2}+2\left(3+\bar{\mu}^{2}\right) x-2=0,
$$

which has roots $z_{0}=\left(1 /\left(1+\bar{\mu}^{2}\right), 1 /\left(1-\bar{\mu}^{2}\right)\right)$ and

$$
z_{5,6}=\left(\left(2 \pm\left(2\left(1-\bar{\mu}^{2}\right)\right)^{1 / 2}\right) /\left(1+\bar{\mu}^{2}\right), \mp\left(2\left(1-\bar{\mu}^{2}\right)\right)^{1 / 2} /\left(1-\bar{\mu}^{2}\right)\right) .
$$

Clearly, $z_{5}$ is not allowable, since $0<y<2$. We now show that $z_{6}$ lies in Region I for all $\bar{\mu}$ such that $0<\bar{\mu}<1$. From (3.5), we note that $T(0)-T(\bar{\mu})=-\bar{\mu}^{2} g(x, y)$, where

$$
g(x, y)=x^{2}\left(y^{2} \bar{\mu}^{2}+y^{2}+1\right)+x\left(2 y-4 y^{2}\right)+y^{2} .
$$

Since for all $\bar{\mu}$ such that $0<\bar{\mu}<1$,

$$
g\left(z_{6}\right)=4 \bar{\mu}^{2}\left(1-\bar{\mu}^{2}\right)^{-1}\left(1+\bar{\mu}^{2}\right)^{-2}\left[\bar{\mu}^{2}-3+2\left(2\left(1-\bar{\mu}^{2}\right)\right)^{1 / 2}\right] \leqq 0,
$$

we have $T(0) \geqq T(\bar{\mu})$ and $z_{6}$ lies in Region I rather than in Region II.

We now show that $z_{1}$ is an extraneous root. Since $\Gamma^{1 / 2}\left(z_{1}\right)=\bar{\mu}\left(1+\bar{\mu}^{2}\right)^{1 / 2}$ and $\Gamma^{1 / 2}\left(z_{0}\right)=\bar{\mu} /\left(1+\bar{\mu}^{2}\right)^{1 / 2}, \Gamma^{1 / 2}\left(z_{0}\right)<\Gamma^{1 / 2}\left(z_{1}\right)$. In fact, $\Gamma^{1 / 2}$ at $z_{0}$ is less than $\Gamma^{1 / 2}$ at any point of the boundary of Region II. This follows since the minimum point of the boundary of Region II is at $\left(4 /\left(5+\bar{\mu}^{2}\right), 4 /\left(3-\bar{\mu}^{2}\right)\right)$ where $\Gamma^{1 / 2}<1$. Note from Young [11], [12] that $1 \leqq S\left(\mathscr{L}_{x, y}\right) \leqq\left\|\mathfrak{L}_{x, y}\right\|_{D^{1 / 2}}$ for $(x, y)$ on the boundary of the square $0 \leqq x \leqq 2,0 \leqq y \leqq 2$. Hence, the point $z_{0}=\left(1 /\left(1+\bar{\mu}^{2}\right), 1 /\left(1-\bar{\mu}^{2}\right)\right)$ minimizes the $D^{1 / 2}$-norm when restricted to Region II. 
We now determine whether $z_{0}$ lies in Region I or Region II for all values of $\bar{\mu}$ such that $0<\bar{\mu}<1$. From (3.23), we have $g\left(z_{0}\right)=1-3 \bar{\mu}^{2}$, so that $z_{0}$ lies in Region II, if $0<\bar{\mu}<(1 / 3)^{1 / 2}$, and in Region I, if $(1 / 3)^{1 / 2}<\bar{\mu}<1$. Clearly, $z_{0}$ lies on the boundary $C$, if $\bar{\mu}=(1 / 3)^{1 / 2}$.

In conclusion, we have, if $0<\bar{\mu} \leqq(1 / 3)^{1 / 2}$, then the optimum relaxation parameters for the $D^{1 / 2}$-norm lie in Region II and are $x=1 /\left(1+\bar{\mu}^{2}\right)$ and $y=1 /\left(1-\bar{\mu}^{2}\right)$. If $(1 / 3)^{1 / 2} \leqq \bar{\mu}<1$, then the optimum values for the virtual $D^{1 / 2}$-norm lie on the boundary $C$ and they are $x=4 /\left(5+\bar{\mu}^{2}\right)$ and $y=4 /\left(3-\bar{\mu}^{2}\right)$. Notice that when $\bar{\mu}=(1 / 3)^{1 / 2}$ we have $1 /\left(1+\bar{\mu}^{2}\right)=4 /\left(5+\bar{\mu}^{2}\right)$ and $1 /\left(1-\bar{\mu}^{2}\right)=4 /\left(3-\bar{\mu}^{2}\right)$. From Theorem 3.1, the proof of Theorem 3.2 follows.

Fig. 3.2 illustrates the behavior of the virtual $D^{1 / 2}$-norm for $\bar{\mu}^{2}=1 / 3$. Notice that the virtual $D^{1 / 2}$-norm is a positive function of $\omega$ and $\omega^{\prime}$ which is less than or equal to unity in Region I but greater than unity in part of Region II.

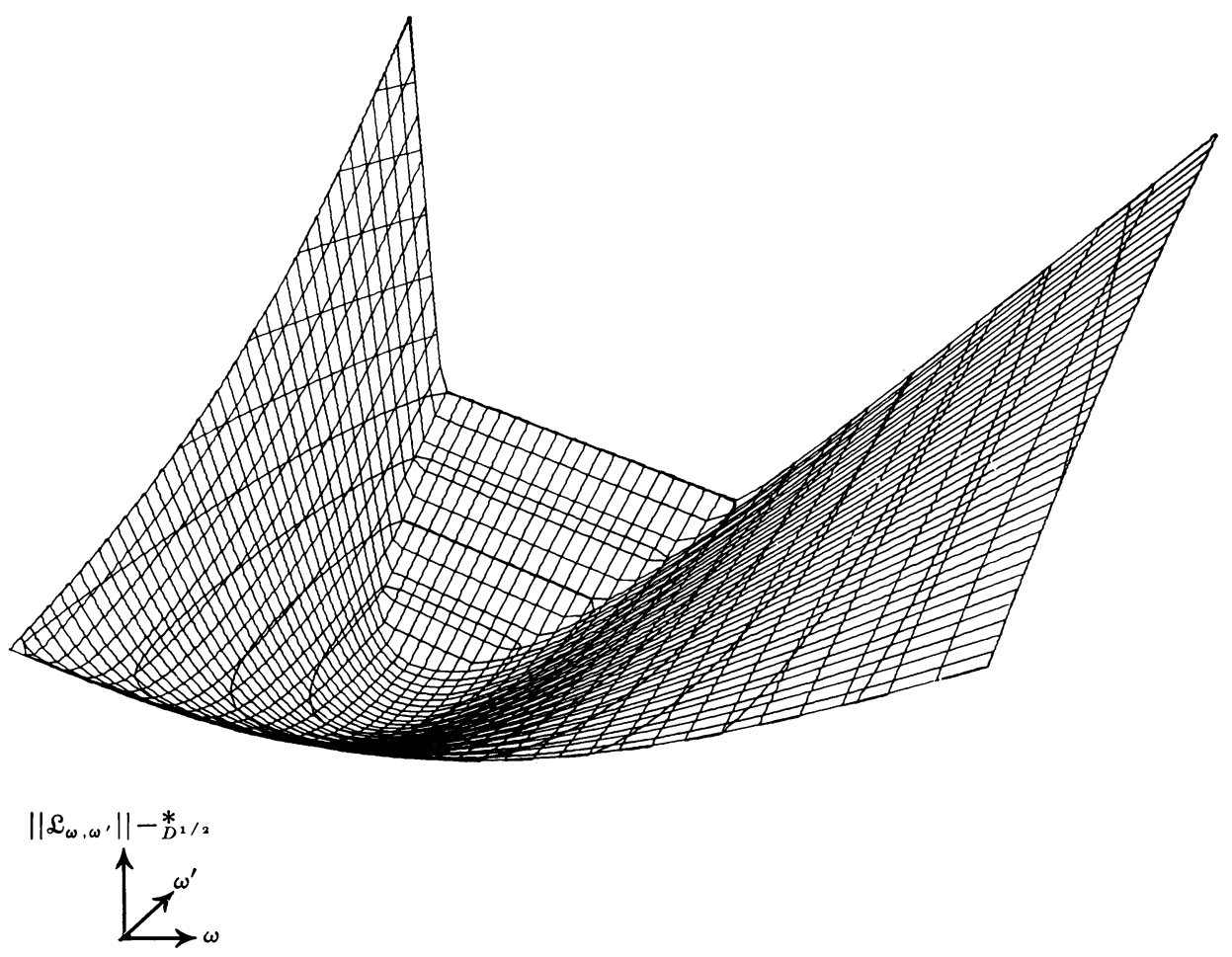

FIGURE 3.2. Virtual $D^{1 / 2}$-norm of $\mathfrak{L}_{\omega, \omega}$.

4. Acknowledgements. The authors wish to thank the staff of the Computation Center of The University of Texas at Austin for assistance in obtaining the perspective graphs.***

*** D. M. Phillips (and E. M. Greenawalt), "Perspective representation of functions of two variables, with overlaid contours," J5 UTEX PERSPEC, Computation Center, The University of Texas at Austin, 1972. 
Center for Numerical Analysis

The University of Texas at Austin

Austin, Texas 78712

1. R. De Vogelaere, “Over-relaxations," Notices Amer. Math. Soc., v. 5, 1958, p. 147. Abstract \#539-53.

2. LouIs W. EHRLICH, "Solving the biharmonic equation as coupled finite difference equations," SIAM J. Numer. Anal., v. 8, 1971, pp. 278-287.

3. Louis W. EHRLICH, "Coupled harmonic equations, SOR, and Chebyshev acceleration," SIAM J. Numer. Anal. (To appear.)

4. L. K. MCDowell, Variable Successive Overrelaxation, Report \#244, Department of Computer Sciences, University of Illinois, Urbana, Ill., 1967.

5. P. J. TAYLOR, "A generalisation of systematic relaxation methods for consistently ordered matrices," Numer. Math., v. 13, 1969, pp. 377-395. MR 41 \#4788.

6. David R. KINCAID, An Analysis of a Class of Norms of Iterative Methods for Systems of Linear Equations, Ph.D. Dissertation, University of Texas at Austin, 1971.

7. David R. KINCAID, A Class of Norms of Iterative Methods for Solving Systems of Linear Equations, CNA-24, Center for Numerical Analysis, University of Texas at Austin, 1971; revised form, Numer. Math. (Submitted for publication.)

8. David R. Kincaid \& David M. Young, The Modified Successive Overrelaxation Method with Fixed Parameters, CNA-33, Center for Numerical Analysis, University of Texas at Austin, 1971.

9. David M. Young, Mary F. Wheeler \& James A. Downing, "On the use of the modified successive overrelaxation method with several relaxation factors," Proc. of IFIP 65, edited by W. A. Kalenich, Spartan Books, Washington, D. C., 1965, pp. 177-182.

10. David M. Young \& David R. Kincaid, Norms of the Successive Overrelaxation Method and Related Methods, TNN-94, Computation Center, University of Texas at Austin, 1969.

11. David M. Young, Convergence Properties of the Symmetric and Unsymmetric Successive Overrelaxation Methods and Related Methods, TNN-96, Computation Center, University of Texas at Austin, 1969; revised version, Math. Comp., v. 24, 1970, pp. 793-807.

12. David M. Young, Iterative Solution of Large Linear Systems, Academic Press, New York, 1971. 